\title{
A New Cortical Surface Parcellation Model and Its Automatic Implementation
}

\author{
Cédric Clouchoux ${ }^{1}$, Olivier Coulon ${ }^{1}$, Jean-Luc Anton ${ }^{2}$, \\ Jean-François Mangin ${ }^{3}$, and Jean Régis ${ }^{4}$ \\ ${ }^{1}$ Laboratoire LSIS, UMR 6168, CNRS, Marseille, France \\ ${ }^{2}$ Centre d'IRM fonctionnelle de Marseille, Marseille, France \\ ${ }^{3}$ Equipe UNAF, SHFJ, CEA/DSV, Orsay, France \\ ${ }^{4}$ INSERM U751, Marseille, France
}

\begin{abstract}
In this paper, we present an original method that aims at parcellating the cortical surface in regions functionally meaningful, from individual anatomy. The parcellation is obtained using an anatomically constrained surface-based coordinate system from which we define a complete partition of the surface. The aim of our method is to exhibit a new way to describe the cortical surface organization, in both anatomical and functional terms. The method is described together with results applied to a functional somatotopy experiments.
\end{abstract}

\section{Introduction}

Understanding the organisation of the human cortex has been a very challenging field of research for the neuroimaging community in the last few years. One of the major challenges is to find relationships between anatomy and function. In this framework, considering the cortex as a 2D-surface has been shown to be a very interesting and efficient way to analyze and to try to understand the cortical organisation. Whereas many representations and models of the cortical anatomy have been proposed, the interaction between anatomy and functions are a lot harder to model. In 8 , cortical maps are described, which suggest a specific organisation of the brain functions in relation with the cortex anatomy. Besides, different methods to represent and to label the human brain cortex have been presented previously $5[12[3$. An interesting approach consists in parcellating the cortical surface, in order to identify regions, patches or gyri, which may have both anatomical and functional homogeneity. This kind of approach offers solutions to describe the cortical surface by overcoming the sulcal variability. Few methods describe a way to automatically define the parcels [14, although different nomenclatures can be used. In [4, the cortical surface is labelled via probabilistic information estimated from a manually labelled training set. In [1, sulci are considered as indicators of the meeting lines buried in the fold's depth between two neighboring gyri. A limit of this method is that the model of sulci does not define all the limits of the gyri, which implies some technical problems and instability. In this paper, we propose a new approach to automatically define a robust gyri-based parcellation of the cortical surface. 
Our method relies on stable anatomical features, the sulcal roots [10], and their known geometric organisation on the cortical surface. Those features are used to first define an individual surface-based coordinate system as described in [2]. From this parameterization, we define a complete partition of the cortex in gyri defined by anatomically stable and functionally meaningful iso-coordinate axis. We aim at exhibiting reproducible gyri with an anatomical/functional homogeneity. The paper is organized as follow: in section 2, we present the cortical anatomy theories underlying our method. Section 3 presents the method itself. An experimental protocol is presented in section 4, and results and discussion about the method are proposed in section 5 .

\section{Anatomical Background and Parcellation Model}

Inter-subject variability is a major problem in studying human brain. One reason for this variability, especially the pattern of sulci, is thought to be the result of the cortex folding process. Several representations of the cortex try to understand the sulci organisation over the cortical surface [1310 7 14 12 5]. A geometric model is proposed in [14, suggesting an orthogonal organization of the main sulci. In [10, an original approach is proposed to explain the sulci organisation: the sulcal roots. The main idea of this new model is the spatial stability of deeply buried subparts of sulci, corresponding to the first folding location during antenatal life. Moreover, this model proposes that these entities are naturally organised, according to two major orthogonal directions. This does not only provide an anatomical model of the sulci organization over the cortical surface, but also gives a functional meaning to it. The cerebral cortex is divided into anatomically and functionally distinct areas, forming a species-specific area map across the cortical sheet. The protomap model 9] proposes that cells of the embryonic vesicle carry intrinsic programs for species-specific cortical regionalization [8]. Hence, the protomap stability across individuals should imply stability of the folding process during gestation [15. This observation, assuming that the deeply buried cortex at the adult stage correspond to the places where the folding process begins at the foetal stage, leads us to hypothesize the existence of prosulcal and progyral maps embedded in the protomap. This implies that sulcal roots related folds are bound to occur somewhere into this map related to boundaries between cortical areas. In [16], it is shown that some boundaries between architectonic maps do clearly correspond to cortical folding. Admitting that sulcal roots are closely related to first folding locations raises the fact that aligned sulcal roots are thought to describe axes delimiting functional cortical areas. For instance, an axis going through several sulcal roots, from the Central Sulcus to the frontal cortex describes a modal gradient. Another example would be an axis, also going through several sulcal roots, along the Central Sulcus, describing a vertical somatotopic gradient. Hence, sulcal roots are thought to be crucial information for both anatomical and functional organization of the cortical surface. In 1], correlation between sulci-based and gyri-based representations has been established. In our approach, a meaningful parcellation can be 


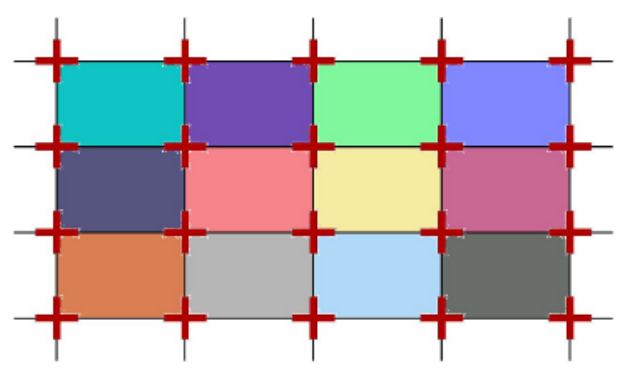

Fig. 1. Sulcal roots (in red) delimiting different parcels

raised using axes described by several sulcal roots alignments. Figure 1 shows a schematic description of a parcel. Following the sulcal roots model, an axis going through several sulcal roots has a functional meaning, separating 2 different functional regions. Therefore, we define a parcel as the region defined by 4 axes, two meridians and two parallels. In other words, a parcel is a region delimited by four sulcal roots (fig. 1). We then aim at identifying, in a population, similar cortical regions having anatomical and functional homogeneity.

\section{Method}

The parcellation we propose here is based on an anatomically constrained surface parameterization, presented in [2, based on the sulcal roots theory presented above. From the resulting coordinate system, we define a parcellation using some specific axes, corresponding to the alignement of several anatomical markers associated with sulcal roots.

\subsection{Surface-Based Coordinate System}

The first step of the parcellation is to define an individual cortical coordinate system on the white matter/grey matter interface, constrained by a set of anatomical markers. The process is fully described in 2 . The set of anatomical markers was defined by studying a number of brains. From this expertise, a generic set of landmarks was chosen. They are subparts of sulci, corresponding to folds around sulcal roots. For each individual brain, automatic labelling of sulci [1] is performed. Identified markers are then projected on the cortical surface, and each one is attributed a coordinate value. Attention must be given to this step, as it guarantees a homogeneous spreading of the coordinates on the whole surface and minimizes metric distortions. Coordinates are then propagated on the surface using the heat equation, where anatomical markers are used as heat sources. In order to guarantee a better regularity of the coordinate field and minimize distortions of parallel/meridians, the process has been improved in the following way : the "heat equation with constant sources" model has been changed to a heat diffusion process with an additional data-driven term that minimizes the 
mean square difference between the coordinate field and the marker coordinates, as defined in equation 1 .

$$
\frac{\partial I(\boldsymbol{r}, t)}{\partial t}=\Delta I(\boldsymbol{r}, t)-\beta(\boldsymbol{r})(I(\boldsymbol{r}, t)-C(\boldsymbol{r})),
$$

where $I(\boldsymbol{r}, t)$ stands for the considered coordinate at node $\boldsymbol{r}$ at time $t . C(\boldsymbol{r})$ is a function that equals 0 everywhere except at the markers location where it equals the marker coordinate. $\beta(\boldsymbol{r})$ is a weight function that equals 0 if $C(\boldsymbol{r})=0$ (no marker) and a constant value $\beta$ if $C(\boldsymbol{r}) \neq 0$. The resulting coordinate system is illustrated in figure 2. Iso density lines of the coordinates system follows the geometry of the surface, and locally complies with the anatomical constraints.
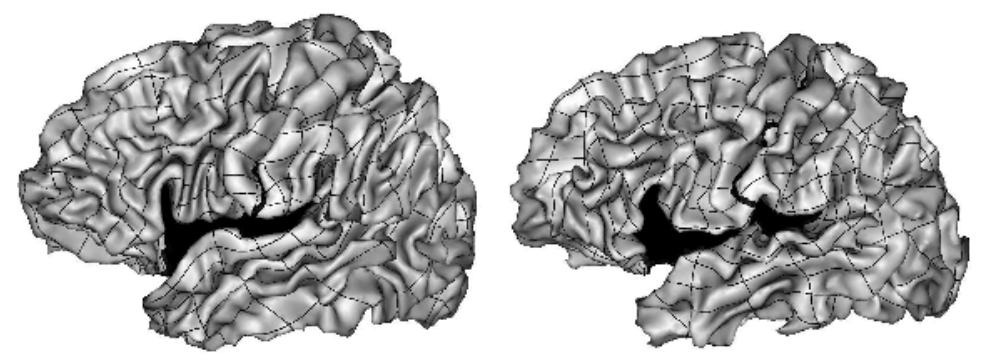

Fig. 2. Resulting coordinate systems on 2 different brains (All visualizations made with free package Brainvisa - http://brainvisa.info)

\subsection{Parcellation}

Isodensity lines going through the predefined anatomical markers are very specific axes. First, as they are constrained to fit with the sulcal roots organization, they are reproducible through individuals 2. Second, they are believed to be functionally meaningful, since they represent the alignement of several sulcal roots. Hence, we can use those sulcal roots related axes to delimit parcels over the entire cortical surface. An example of such parcellation is shown in figure 3. Parcels described here are orthogonally organised, following the sulcal roots scheme (fig. 4), and neighborhood relationships between gyri are conserved.

\section{Experiments}

We tested our parcellation method on a functionnal experiment on a set of mature non pathological brains. The method was applied on 10 right-handed healthy volunteers who were part of a somatotopy experiment. Individual tstatistic maps corresponding to right motor activations of the foot, elbow, little finger, index finger, thumb, and tongue were projected on individual left brain hemisphere. Maxima corresponding to primary motor activations were then extracted, and plotted on each brain surface (fig. 5). 


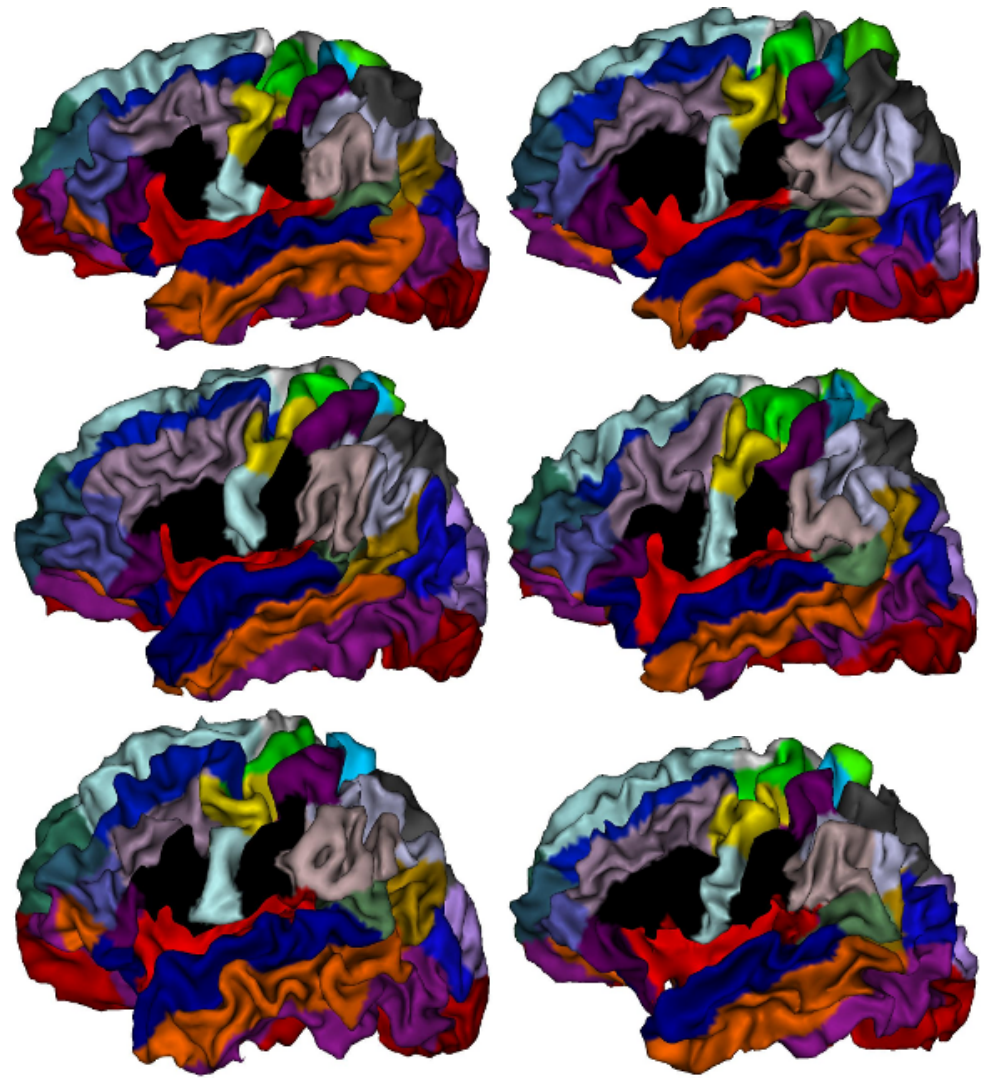

Fig. 3. Results of the parcellation on six different brains

\section{Results and Discussion}

What we expect from our first experiment is a reproductibility of the parcellation across individuals, and a good fit with individual anatomical features. We also expect the primary motor activations involving different parts of the body to be located in different parcels. For instance, the parallel going through the Central Sulcus, the Interparietal Fissure and the Temporal Superior Sulcus describes a modal gradient. Moreover, it is known to delimitate, at the location of these three sulci, a separation of functional process involving the face and process involving lower parts of the body. Therefore, we expect such a separation at the somatotopic level in the primary motor areas along the precentral gyrus. Similarly, we would expect a separation between the foot and higher parts of the body along the same gyrus.

Figure 3 shows the resulting parcellation on 6 different brains. A first observation is that the different parcellations are respectful of each individual anatomy, 


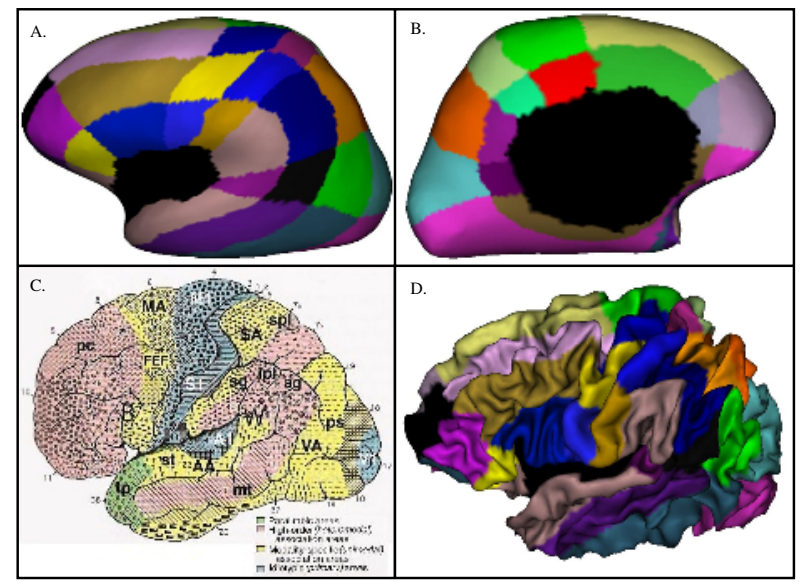

Fig. 4. (A and B) Resulting parcellation on an inflated cortical surface. (C) A cytoarchitectonic and functional parcellation of the human cerebral cortex [7] , compared to (D) our automatic parcellation.

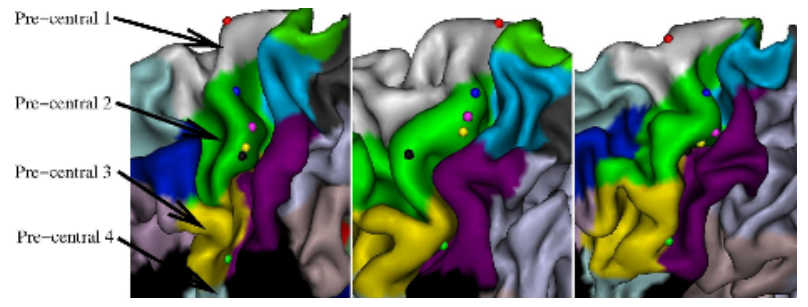

Fig. 5. Plotting of right motor activations on 3 parcellated brains. Activations colors: red $=$ foot, blue $=$ elbow, pink=little finger, yellow $=$ index finger, black=thumb, green $=$ tongue.

and complies with the cortex geometry. Moreover, parcellation is stable across individuals. All the regions are present in every subject, with a stable relationship scheme between the different parcels. Figure 4 (A,B) shows the parcels organization on an inflated brain. It is interesting to point out the similarity between the cytoarchitectonic and functional scheme presented in [6] and the parcellation model we get (see figure 4).

Figure 5 shows the plotting of right motor activations on 3 brains. The usual pre-central gyrus is here divided in 4 parcels, called pre-central gyrus $1,2,3$ and 4 . On 10 subjects, the results of our experiment were : all the foot activations were located in pre-central gyrus 1 . On 40 activations of the arm (elbow and 3 fingers), 37 are located in gyrus pre-central 2, 1 at the limit between pre-central gyri 1 and 2, and 2 at the limit between pre-central gyri 2 and 3.7 tongue activations were in the precentral gyrus 3 , the 3 others beeing located 
in the pre-central gyrus 4 . We therefore observe that the delimitation induced by the parallel Central Sulcus/Interparietal Fissure/Temporal Superior Sulcus systematically separates activations of the face from activations of the rest of the body. Foot activations are also dissociated from the rest of the body. These results seem to show the expected functional specialization of our parcellation model.

It is interesting to note that our parcellation provides a higher level of description than more usual models. However, the set of anatomical markers used to constrain our coordinate system (and therefore our parcellation scheme) is the same than the one used in [1] to directly define gyrus. Therefore, it seems natural that by merging our parcels, we can recover the gyri decribed in [1]. For instance, the pre-central gyrus can be recovered by merging pre-central gyri 1 , 2, 3 and 4 described in figure 3. Figure 6 shows an example of this. By doing so, we get a better definition than in the parcellation presented in [1], which uses anatomical features to define 2 borders of a gyri, while the 2 other borders are defined by competing evolution on the surface. This induces problems: unstable neighborhood relationships between gyri, and unstable delimitation of some gyri, whereas with the method presented here, all parcels are directly defined by anatomical information and are therefore more stable.
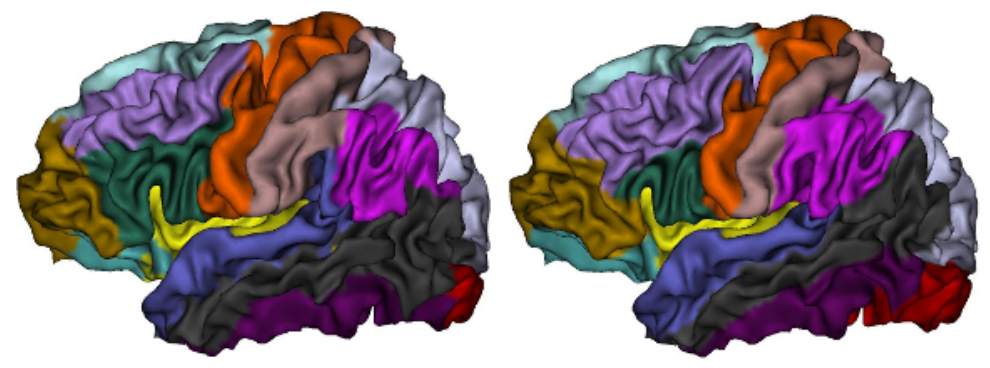

Fig. 6. Recovering a previous gyral description. (left) Gyral parcellation presented in 1]. (right) Gyral parcellation obtained by merging parcels produced by our method.

\section{Conclusion}

In this paper we presented a method to automatically parcellate the cortical surface in regions functionally meaningful, using an anatomically constrained surface-based coordinate system. The parcellation is reproducible through individuals, and primary activations involving different parts of the body are located in different gyri, which shows that our method is both anatomically and functionally relevant. The method has many potential neuroscience applications, such as cortex morphometry studies, functional localization and inter-subject functional and anatomical correlation studies. 


\section{References}

1. A. Cachia, J.-F. Mangin, D. Rivière, D. Papadopoulos-Orfanos, F. Kherif, I. Bloch, and J. Régis. A generic framework for parcellation of the cortical surface into gyri using geodesic voronoi diagrams. Medical Image Analysis, 7(4):403-416, 2003.

2. C. Clouchoux, O. Coulon, D. Riviere, A. Cachia, J-F. Mangin, and J. Regis. Anatomically constrained surface parameterization for cortical localization. In J. Duncan and G. Gerig, editors, LNCS - Medical Image Computing and ComputerAssisted Intervention - MICCAI 2005: 8th International Conference, volume 3750, pages 344-351. Springer-Verlag Berlin Heidelberg, octobre 2005.

3. D.C. Van Essen and H.A. Drury. Structural and functional analyses of human cerebral cortex using a surface-based atlas. The Journal of Neuroscience, 17(18):7079 7102, 1997.

4. B. Fischl, A. van der Kouwe, C. Destrieux, E. Halgren, Florent Sgonne, D.H. Salat, E. Busa, L.J. Seidman, J. Goldstein, D. Kennedy, V. Caviness, N. Makris, B. Rosen, and A.M. Dale. Automatically parcellating the human cerebral cortex. Cerebral Cortex, 14:11-22, 2004.

5. J.-F. Mangin, V. Frouin, I. Bloch, J. Régis, and J. López-Krahe. From 3D magnetic resonance images to structural representations of the cortex topography using topology preserving deformations. Journal of Mathematical Imaging and Vision, 5:297-318, 1995.

6. M.-M. Mesulam. Principles of Behavioral and Cognitive Neurology, Second Edition. Oxford University Press, New York, 2000.

7. M. Ono, S. Kubik, and C. Abernathey. Atlas of the cerebral sulci. Thieme Medical Publishers, Inc., New York, 1990.

8. P. Racik. Neurobiologie. neurocreationism - making new cortical maps. Science, 294:1011-1012, 2001.

9. P. Rakic. Specification of cerebral cortical areas. Science, 241:170-176, 1988.

10. J. Régis, J.F. Mangin, T. Ochiai, V. Frouin, D. Rivière, A. Cachia, M. Tamura, and Y. Samson. Sulcal roots generic model: a hypothesis to overcome the variability of the human cortex folding patterns. Neurol Med Chir, 45:1-17, 2005.

11. D. Rivière, JF. Mangin, D. Papadopoulos-Orfanos, J.M. Martinez, V. Frouin, and J. Régis. Automatic recognition of cortical sulci of the human brain using a congregation of neural networks. Medical Image Analysis, 6(2):77-92, 2002.

12. P.M. Thompson, C. Schwartz, and A.W. Toga. High-resolution random mesh algorithms for creating a probabilistic 3 -d surface atlas of the human brain. Neuroimage, 3:19-34, 1996.

13. P. Todd. A geometric model for the cortical folding pattern of simple folded brains. J Theor Biol, 97:529 538, 1982.

14. R. Toro and Y. Burnod. Geometric atlas: modeling the cortex as an organized surface. NeuroImage, 20(3):1468-1484, 2003.

15. O. Turner. Growth and development of the cerebral pattern. Man. Arch. Neurol.Psychiatry, pages 1-12, 1948.

16. K. Zilles, A. Schleicher, C. Langemann, K. Amunts, P. Morosan, N. PalomeroGallagher, T. Schormann, H. Mohlberg, U. Brgel, H. Steinmetz, G. Schlaug, and P.E. Roland. Quantitative analysis of sulci in human cerebral cortex: Development, regional heterogeneity, gender difference, asymetry, intersubject variability and cortical architecture. In Human Brain Mapping, volume 5, pages 218-221, 1997. 